\title{
Molecular dynamic insight into the ethanol effect on Tretinoin drug delivery through carbon nanotubes
}

\author{
Maryam Ghadamgahi · Davood Ajloo
}

Received: 12 October 2013/Accepted: 13 December 2013/Published online: 5 March 2014

(C) The Author(s) 2014. This article is published with open access at Springerlink.com

\begin{abstract}
Carbon nanotubes as potential material for drug delivery can pass through cell walls, transfer, and liberate drugs in particular tissues. The goal of this article is to survey the insertion of an anticancer drug called Tretinoin into CNT $(10,7)$ and $\mathrm{CNT}(8,5)$. All computations are surveyed by molecular dynamics simulation in various temperatures $(270$ and $310 \mathrm{~K})$ and in water solution and ethanol co-solvent. $0.2 \mathrm{M}$ ethanol is studied that is more than normal range of blood alcohol content. The molecular dynamic simulation results show that the insertion of Tretinoin into the carbon nanotube in a water solute environment could be related to the diameter of the nanotube and in the flow of the waters via hydrophilic interactions. Insertion of drug into a CNT $(10,7)$ is observed in the presence of water at $310 \mathrm{~K}$ and ethanol inhibits encapsulation. The results of structure parameters and free energy of interaction indicate that the energy of nanotube and Tretinoin decreases after the encapsulation in water, and increases in ethanol co-solvent. The results also show that by increasing initial temperature, heat capacity increases.
\end{abstract}

Keywords Molecular dynamic simulation - Carbon nanotube $\cdot$ Tretinoin $\cdot$ Co-solvent

$\begin{array}{ll}\text { Abbreviations } \\ \text { CNTs } & \text { Carbon nanotubes } \\ \text { MD } & \text { Molecular dynamic }\end{array}$

M. Ghadamgahi · D. Ajloo

School of Chemistry, Damghan University, Damghan, Iran

e-mail: m_ghadamgahi@std.du.ac.ir

D. Ajloo $(\bowtie)$

Faculty of Chemistry, College of Science, University of Tehran,

Tehran, Iran

e-mail: ajloo@du.ac.ir
EtOH Ethanol
RMSD Root-mean-square derivation
COM Center of mass
RDF Radial distribution function

\section{Introduction}

Nowadays, in the universe of new medicine, carbon nanotubes have experimentally showed their capacity in passing through the cell shell. Accordingly, scientists believe that they can apply them in releasing active drug molecules in the cell, particularly in the most sensitive and essential molecules for special diseases such as cancer and AIDS. The physical and chemical nature of these nanotubes has been studied by many scientists to prepare this material for such a significant task [1]. The major aim of developing nanocarrier drug delivery systems is to enhance the therapeutic effect or reduce toxicity of therapeutically active materials. This is conventionally achieved using cylindrical molecules made of carbon atoms such as carbon nanotubes (CNTs) [2, 3].

Skin cancer is the most common form of human cancer. As a result, many drugs have been developed to treat skin cancer. In addition, there are very few demonstrable biochemical differences between cancerous cells and normal cells. For this reason, the effectiveness of many anti-cancer drugs is limited by their toxicity to normal rapidly growing cells in the intestinal and bone marrow areas. A final problem is that cancerous cells which are initially suppressed using a specific drug may develop a resistance to that drug. For this reason, cancer treatments may consist of using several drugs in combination for varying lengths of time [4-6]. The anti-cancer drug that was selected in this work is Tretinoin that is an important regulator of cell 
reproduction, proliferation, and differentiation and is used to treat acne and photo-damaged skin and to manage keratinization disorders such as ichthyosis and keratosis follicularis [7-9].

A number of studies reveal that drug delivery property of the drugs depends on solvent environment and temperature [10]. The interaction of a nanotube with an anticancer agent named 2-(2-amino 6,7-dimethyl Pteridine 4-ylamino) has been investigated in methanol, ethanol and water by quantum mechanics and semi-empirical methods. Results revealed that these composites are more stable in water than the other solvents [11].

Kiruta et al. [12] have previously studied doxorubicin insertion into carbon nanotube and concluded that for nanotube with small diameter $d<12.5$, doxorubicin is located outside the nanotube and for larger diameters; the drug is located inside the tube. In the other case, the encapsulation of Levothyroxine as a drug and carbon nanotube $(9,0)$ is investigated by Dalili Mansour [13]. It is proved that there is an optimal tube size which provides the most effective encapsulation for a given drug molecule, and the encapsulation in the nanotubes is influenced by drug diameter. Consequently, two diameters of $(10,7)$ and $(8,5)$ of CNT is selected and examined by MD simulation which are the smallest optimum size of CNT for Tretinoin encapsulation.

Thus by emphasizing the strong effect of Tretinoins as known drugs of skin anti-cancer and aiming to decrease the toxicity of them, we thought about target therapy in which nanotube with optimum size is applicable as a carrier for these drugs. This paper is also concentrated on the free energy of interaction between CNT and Tretinoin. Furthermore, the effects of co-solvents like ethanol $(\mathrm{EtOH})$ on drug insertion at two temperatures and two concentrations have been studied and structure parameters and free energies obtained by means of molecular dynamics simulation have been compared.

\section{Materials and methods}

Molecular dynamics

In this work, interaction of carbon nanotubes with Tretinoin drug is investigated. The structure of Tretinoin and EtOH was drawn using HyperChem 7 software [14]. These structures were pre-optimized with molecular mechanics force field $(\mathrm{MM}+)$ and their final geometries were obtained with the semi-empirical AM1 method [15]. Force field parameters of them were generated using PRODRG2 server [16]. A series of uncapped chiral carbon nanotubes with diameters $(10,7)$ and $(8,5)$ were generated using the TubeGen 3.4 Online server (web-interface, http://turin.nss.udel.edu/

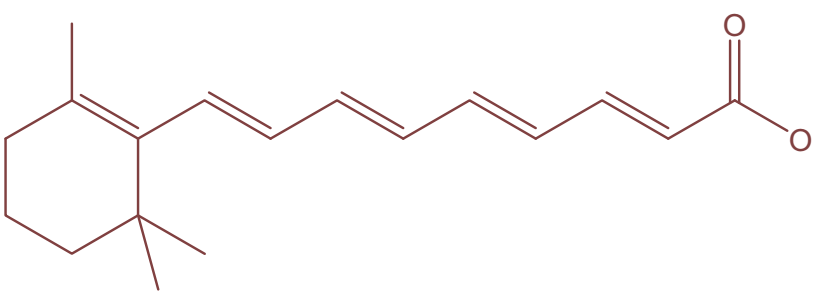

Scheme 1 Structure of skin anticancer drug (Tretinoin)

research/tubegenonline.html) [17]. GROMOSE96 53a6 force field was used to model atomic interactions [18]. The parameters of the CNT were taken from this force field [19], where the atom type $\mathrm{CA}$ was chosen to represent the aromatic carbon atoms. A general comment on the applicability of this force field to CNT can be found elsewhere [20]. No external forces were applied to the Tretinoin for entering into the nanotubes. As initial configurations, CNT and drug were aligned along the nanotube axis and separated sufficiently far away $(>3 \mathrm{~nm}$ ) to minimize the effect of starting orientations. Then, water molecules were randomly added into the simulation box with SPC216 water as solvent [21]; the charge of the system was zero and its dynamics was simulated for $16 \mathrm{~ns}$. Molecular dynamics simulation was performed using GROMACS software version 3.3.1 [22]. In all simulations, the temperature and the pressure were kept close to the intended values (270 and $310 \mathrm{~K}$ and 1 bar) using the NoseHoover and Parrinello-Rahman algorithms, respectively [22]. Molecular dynamic velocity Verlet integration algorithm was used and all bond lengths, including those to hydrogen atoms, were constrained using LINCS algorithm. Electrostatic interactions between charged groups within $0.9 \mathrm{~nm}$ were calculated explicitly, while long-range electrostatic interactions were calculated using the ParticleMesh Ewald (PME) method [23]. Lennard-Jones and van der Waals interactions were calculated with a $0.9 \mathrm{~nm}$ range cutoff. A time step of $2 \mathrm{fs}$ was used for the integration of motion equation. The type of algorithm to search neighbors was Grid. Co-solvent of EtOH solvated in the cellular solutions can have profound effects on the studied systems. Consequently, $0.2 \mathrm{M} \mathrm{EtOH}$ was added to the simulation box. Heat capacities, kinetic and potential energies, diffusion coefficient, free energies and other parameters were compared for studied systems. The other aim of this paper is to compare results of systems within two temperatures to offer an insight for our own theoretical work and into drug delivery ability under different temperature conditions. Scheme 1 shows the structure of Tretinoin.

All of the simulations were equilibrated by $1 \mathrm{~ns}$ with position restraints on the Tretinoin and nanotube to allow for the relaxation of the solvent/co-solvent molecules. After equilibration, the molecular dynamic runs were 
Table 1 Summary of studied systems and the duration of each system was $3 \times 16 \mathrm{~ns}$

\begin{tabular}{|c|c|c|c|c|c|}
\hline No & $\mathrm{CNT}$ & $\begin{array}{l}\text { Solvent/ } \\
\text { co-solvent }\end{array}$ & $\begin{array}{l}\text { No. } \\
\text { water }\end{array}$ & $\begin{array}{l}\text { Con. co- } \\
\text { solvent (M) }\end{array}$ & $T(\mathrm{~K})$ \\
\hline 1 & $\begin{array}{l}\text { Tretinoin-CNT } \\
\quad(8,5)\end{array}$ & Water & 4,382 & 0 & 270 \\
\hline 2 & $\begin{array}{l}\text { Tretinoin-CNT } \\
\quad(8,5)\end{array}$ & & 4,382 & 0 & 310 \\
\hline 3 & $\begin{array}{l}\text { Tretinoin-CNT } \\
\quad(8,5)\end{array}$ & Water/EtOH & 4,284 & 0.2 & 270 \\
\hline 4 & $\begin{array}{l}\text { Tretinoin-CNT } \\
\quad(8,5)\end{array}$ & & 4,284 & 0.2 & 310 \\
\hline 5 & $\begin{array}{l}\text { Tretinoin-CNT } \\
(10,7)\end{array}$ & Water & 5,271 & 0 & 270 \\
\hline 6 & $\begin{array}{l}\text { Tretinoin-CNT } \\
(10,7)\end{array}$ & & 5,271 & 0 & 310 \\
\hline 7 & $\begin{array}{l}\text { Tretinoin-CNT } \\
\quad(10,7)\end{array}$ & Water/EtOH & 5,173 & 0.2 & 270 \\
\hline 8 & $\begin{array}{l}\text { Tretinoin-CNT } \\
\quad(10,7)\end{array}$ & & 5,173 & 0.2 & 310 \\
\hline 9 & Tretinoin & Water & 4,466 & 0 & 270 \\
\hline 10 & Tretinoin & & 4,466 & 0 & 310 \\
\hline 11 & Tretinoin & Water/EtOH & 4,358 & 0.2 & 270 \\
\hline 12 & Tretinoin & & 4,358 & 0.2 & 310 \\
\hline
\end{tabular}

16 ns long. Production runs of all of the systems were obtained three times to check repeatability, and analyses were averaged from all three independent trajectories. A summary of the simulations is listed in Table 1. To calculate binding energy of Tretinoin to CNT with LIE algorithm, 4 system of 9-12 in the absence of nanotube was constructed.

\section{Analysis}

Heat capacity measurement

The simulation trajectories were analyzed using several auxiliary programs implemented into the GROMACS package. In general, a straightforward but difficult method to calculate the heat capacity at constant pressure $\left(C_{\mathrm{P}}\right)$ is to use the trajectory energy fluctuations to determine it directly to compare the stability of structures. From a trajectory, one can determine the trajectory average energy and the enthalpy of each step $i H_{i}$ to determine the heat capacity which has been previously calculated [24]. To calculate heat capacity, total energy $(E)$ and PV choice can be made from the list of energy. Following equations are used to calculate the enthalpy $(H)$ and heat capacity $\left(C_{\mathrm{P}}\right)$ of systems:

$H=E+\mathrm{PV}$
$C_{\mathrm{P}}=\frac{H^{2}-H^{2}}{R T^{2}}$

$\langle H\rangle=\frac{\sum_{V} \sum_{j} H_{i} \mathrm{e}^{-E_{V_{j}} / k T} \mathrm{e}^{-\mathrm{pV} / k T}}{\sum_{V} \sum_{j} \mathrm{e}^{-E_{V_{j}} / k T} \mathrm{e}^{-\mathrm{pV} / k T}}$,

$H$ is the average value of enthalpy, $H_{i}$ is enthalpy of $i$ th state and $k$ is Boltzmann constant. The above equations are valid in equilibrium so potential energies are presented to confirm equilibration state.

Diffusion coefficient

Mean square displacement (MSD) is the average distance that a molecule travels over time. MSD is the movements of molecules away from equilibrium position of atoms within whole simulation process due to inter-molecular forces. To determine the self-diffusion coefficient $D_{A}$ of particle $A$, one can use the Einstein relation of MSD:

$\operatorname{MSD}=R(t)=r_{i}(t)-r_{i}(0)^{2}$

$\lim _{t \rightarrow \infty} r_{i}(t)-r_{i}(0)_{i \in A}^{2}=6 D_{A} t$

Where $r_{i}(t)-r_{i}(0)$ is the (vector) distance, travelled by molecule $i$ over some time interval of length $t$, and the squared magnitude of this vector is averaged over many such time intervals. This quantity is often obtained by averaging over all molecules in the system; by summing $i$ from 1 to $N$ and dividing by $N$. The limiting slope of MSD $(t)$, considered for time intervals sufficiently long for it to be in the linear regime, is related to the self-diffusion constant $D_{A}$ that is obtained from slope of MSD curves vs. time which is divided to 6 [25].

Free energy calculation

Linear interaction energy (LIE) method was used to estimate binding free energies. This semi-empirical method has been proposed by A qvist et al. [26] to calculate absolute and relative binding free energies. In this method, the absolute binding free energy of a ligand is estimated as:

$\Delta G_{\mathrm{bind}}=\alpha\left(V_{\mathrm{bound}}^{\mathrm{el}}-V_{\text {free }}^{\mathrm{el}}\right)+\beta\left(V_{\mathrm{bound}}^{\mathrm{vdw}}-V_{\text {free }}^{\mathrm{vdw}}\right)$,

where $V_{\text {bound }}^{\mathrm{el}}$ and $V_{\text {bound }}^{\mathrm{vdw}}$ are the electrostatic and van der Waals interaction energies between the ligand and the solvated nanotube obtained from a molecular dynamics (MD) trajectory with ligand bound to CNT, and $V_{\text {free }}^{\text {el }}$ and $V_{\text {free }}^{\mathrm{vdw}}$ are the electrostatic and van der Waals interaction 


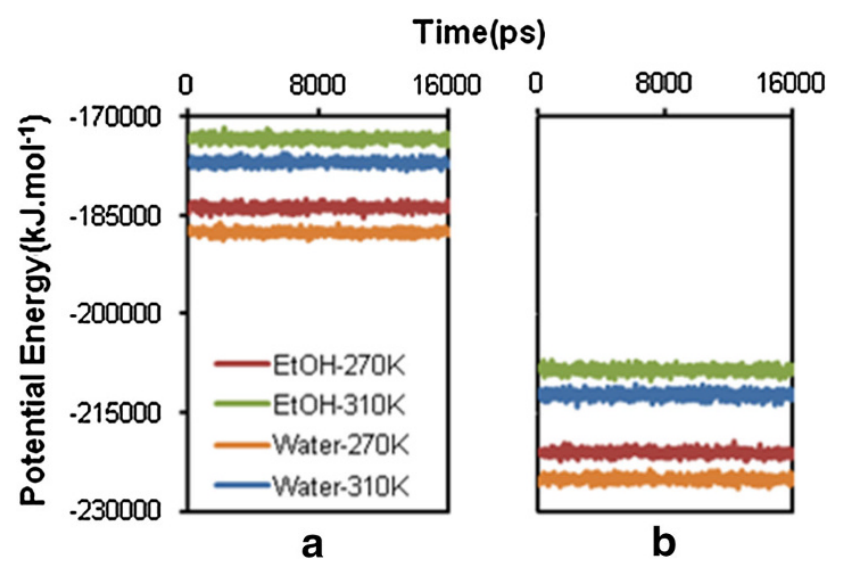

Fig. 1 Potential energy of a CNT (8, 5)-Tretinoin and b CNT $(10,7)$ Tretinoin system in water and co-solvent at two temperatures 270 and $310 \mathrm{~K}$

energies between the ligand and the water from another MD trajectory with the ligand in water. A set of values, $\alpha$ 0.5 and $\beta 0.16$, was found to give good results [26].

Potential of mean force

Potential of mean force (PMF) was calculated between the solutes using:

$\mathrm{PMF}=-K_{\mathrm{B}} T \ln g(\mathrm{r})$

where $K_{\mathrm{B}}$ is the Boltzman constant, $T$ is the simulation temperature, and $g(r)$ is the radial distribution function (RDF) between the solutes [27].

\section{Results and discussion}

\section{System equilibration}

In the first step of analyzing a simulation, it is attempted to determine if the system has reached equilibrium by looking at quantities such as the total and potential energy. Equilibrium can be obtained by putting a restraining potential on the atoms that must be protected to avoid badly placed solvent molecules giving unreasonable kicks to nanotube atom. Here, the potential energy is just reported and other quantities also confirmed equilibrium state (not shown here). Figure 1 shows potential energy of $\operatorname{CNT}(8,5)$-Tretinoin (Fig. 1a) and CNT (10, 7)-Tretinoin (Fig. 1b) after $1 \mathrm{~ns}$ position restraint in water and $\mathrm{EtOH}$ co-solvent at two temperatures. Based on the nearly constant potential energy curves, it appears that the simulation has equilibrated. Both figures confirm more negative potential energy in lower temperatures and in water solution due to less mobility and kinetic energy of system. By comparing obtained potential
Table 2 Enthalpy and obtained heat capacity $\left(C_{\mathrm{p}}\right)$ in different temperatures for different systems

\begin{tabular}{lll}
\hline No & Enthalpy & $C_{\mathrm{p}} /\left(\mathrm{kJ} \mathrm{mol}^{-1} \mathrm{~K}^{-1}\right)$ \\
\hline 1 & $-157,695$ & 374.9874 \\
2 & $-142,737$ & 360.0812 \\
3 & $-154,209$ & 393.2614 \\
4 & $-139,362$ & 374.1397 \\
5 & $-188,744$ & 498.7627 \\
6 & $-170,947$ & 496.5998 \\
7 & $-185,238$ & 446.3232 \\
8 & $-167,409$ & 428.3419 \\
\hline
\end{tabular}

energies in Fig. 1a and b, it becomes clear that by adding carbon atoms of nanotube and an increase in nanotube radius, the system becomes more stable because steric hindrance of the ring and drug decreases. So by increasing CNT size and decreasing temperature, the system becomes more stable in water and the potential energy decreases.

For comparison purpose, heat capacity and enthalpy (heat of formation) were obtained and results were compared. The obtained energies calculated by MD for all of the systems are shown in Table 2 .

It becomes clear that interactions between these drugs and nanotubes have negative heat of formations. So these interactions are exothermic reactions.

Comparison of system 1-4 shows more negative enthalpy energy at lower temperatures and in water solution. This result is also seen for system 5-8. Comparison of enthalpy for system 1-8 shows more negative energy of CNT $(10,7)$. Totally, the lowest values of enthalpy can be obtained when CNT $(10,7)$ carbon nanotube system is in water solution.

Noticeably, in Table 2, we can understand that the decrease in solvent dielectric constant makes the enthalpy values of interaction between CNT and solute molecules (Tretinoin) move toward negative amounts. It reveals that there is interaction between CNT and solute molecules. As it can be seen in Table 2, the energy value for CNT $(10,7)$ /water is lower than the CNT $(8,5)$ and by increasing CNT size, the potential energy becomes lower that confirms results of Fig. 1. So the structure of nanotube becomes more stable by increasing size and the potential energy becomes lower.

By investigating Table 2, it can be seen, by increasing initial temperature in all of the cases kinetic energy and heat capacity decrease. Also it can be seen that by increasing solvent dielectric constant, the heat capacity increases too. Addition of EtOH molecules also decreases system stability and increases heat capacity. These results are in good agreement with previous data [28] and results of Fig. 1.

Furthermore, these systems are more stable in water than other solvents/co-solvents such as EtOH [28]. It is 
Table 3 The dipole moment $(\mu)$, dielectric constant $(\varepsilon)$ and molecular mass of studied co-solvents [18]

\begin{tabular}{llll}
\hline Solvent & Dipole moment (debye) & Dielectric constant & $\begin{array}{l}M_{\mathrm{W}} \\
(\mathrm{g} \mathrm{mol}\end{array}$ \\
\hline EtOH & 1.69 & 24.55 & 46 \\
Water & 1.85 & 80.10 & 18 \\
\hline
\end{tabular}

because of the existence of more hydrogen bonds in water and so the molecule becomes more stable. The other reason for differences of water and $\mathrm{EtOH}$ is the differences of properties presented in Table 3 .

\section{EtOH, temperature and CNT size effect}

Three different snapshots at different times of system in the presence of water and $\mathrm{EtOH}$ at $310 \mathrm{~K}$ are presented in Fig. 2.

The snapshots of the Tretinoin-CNT system shown in Fig. 2 indicated insertion process of Tretinoin into the CNT $(10,7)$ in water solution as compared to results of system in ethanol solution in Fig. 2b. At the beginning of simulation, the Tretinoin came near the tube and at the time of $5 \mathrm{~ns}$ the first molecule of the Tretinoin has begun to enter the nanotube. The drug molecule was totally inserted into the nanotube after $16 \mathrm{~ns}$. All simulations performed in equal situations and results confirm inhibition effect of ethanol from Tretinoin insertion. Other results show no insertion of Tretinoin into CNT at $270 \mathrm{~K}$ and in $\mathrm{CNT}(8,5)$.

Distance curves of CNT-Tretinoin are compared at 310 and $270 \mathrm{~K}$ and in the water solution and $\mathrm{EtOH}$ in Fig. 3. Correspondingly, the center of mass (COM) distance between Tretinoin and CNT $(10,7)$ rapidly decreases with time up to nearly $5 \mathrm{~ns}$ in water. This figure confirms less distance between CNT drug at higher $310 \mathrm{~K}$ and in the presence of water. The comparison of distance for studied systems also reveals $\mathrm{CNT}(8,5)-\mathrm{EtOH}-310 \mathrm{~K}<\mathrm{CNT}(8$, 5)-Water-310 K $<$ CNT(8, 5)-Water-270 K $<$ CNT(8, 5)-EtOH-270 K (Fig. 3a) and CNT (10, 7)-Water$310 \mathrm{~K}<\mathrm{CNT}(10,7)-\mathrm{EtOH}-270 \mathrm{~K}<\mathrm{CNT}(10,7)$-EtOH$310 \mathrm{~K}<\mathrm{CNT}(10,7)$-Water-270 K trend (Fig. 3b). It can be concluded that Tretinoin approaches the CNT $(8,5)$ in EtOH and water at $310 \mathrm{~K}$. The distance between CNT (10, 7)-Tretinoin reduces in water at both temperatures and in EtOH at $270 \mathrm{~K}$, but Tretinoin goes far from CNT $(10,7)$ in
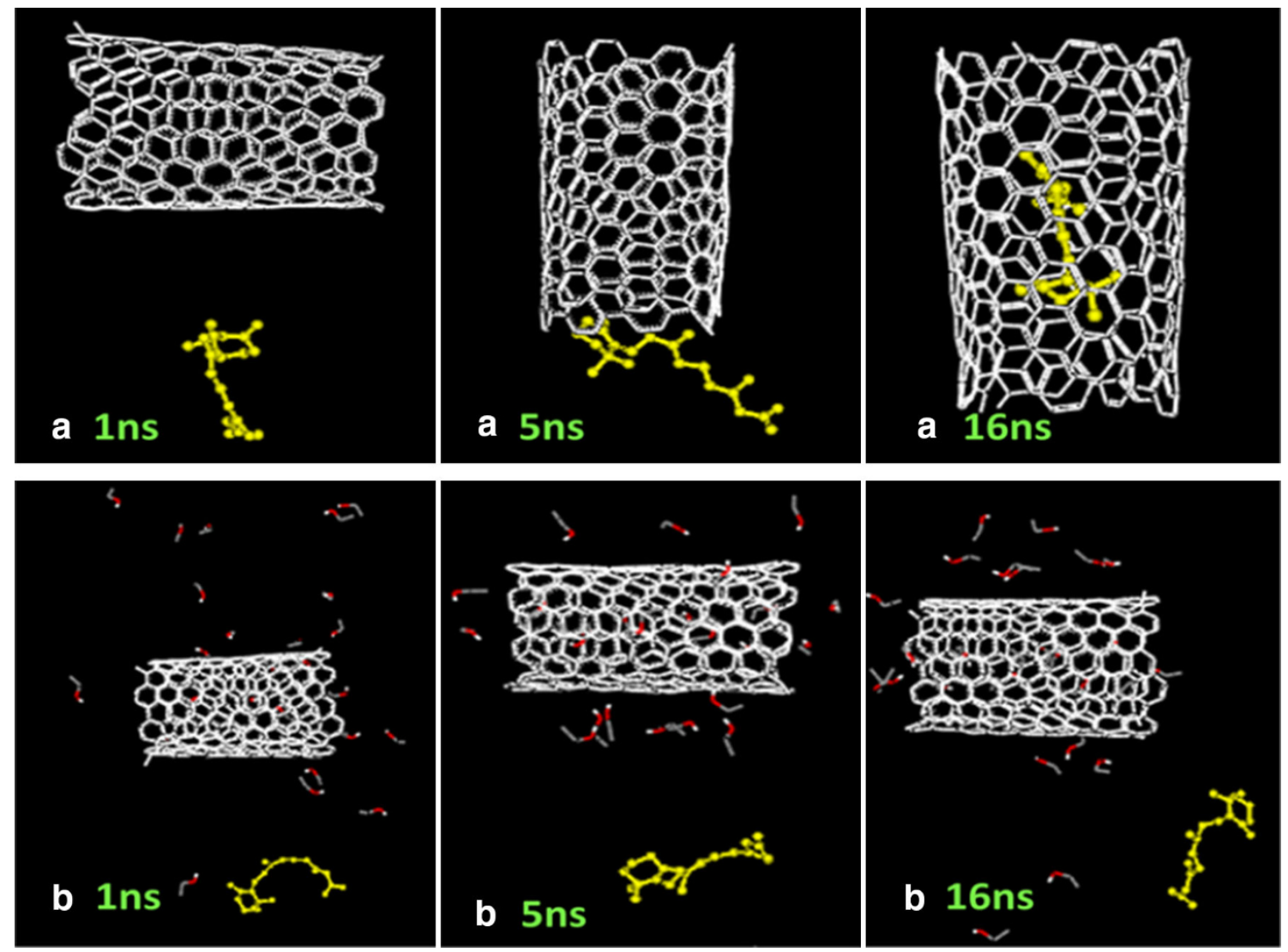

Fig. 2 Snapshot of Tretinoin interacting with a $(10,7)$ carbon nanotube at $1 \mathrm{~ns}$ (after position restraint) 5 and 16 ns in a water solution and b EtOH. Water molecules are removed for clarity 
Fig. 3 Alteration of the center of mass distance of Tretinoin and a CNT $(8,5)$, b CNT $(10,7)$ during the simulation and c distance diagram of system in water at $310 \mathrm{~K}$
$\mathrm{EtOH}$ at $310 \mathrm{~K}$ and the distance increases that confirms results of Fig. 2. Figure 3c shows distance diagram of drug from CNT's axis (CNT $(10,7)$ ) as a function of simulation time, while the averaged projection of the $g(r)$ to the CNT $x$ and $y$ axis (defined in inset Fig. 3c) was also calculated to monitor the movement of the drug in the direction perpendicular to the CNT surface. According to distance result, the Tretinoin has the ability to move freely and locate inside the CNT, and the constant value of distance in $1 \mathrm{~nm}$ reveals drug ability to remain in CNT. The probability of finding the Tretinoin molecule in a direction perpendicular to nanotube $x$ and $y$ axis obtained. Results show one intense peak at $0.8 \mathrm{~nm}$. This peak indicates that drug does not prefer to move along the nanotube $\mathrm{z}$-axis and the favorite motion is $1.4 \mathrm{~nm}$ away from inner surface of nanotube and this result is in good agreement with previous data [29]. Accordingly, all the above mobility data revealed that Tretinoin prefers to coordinate inside the CNT during the whole simulation time of $16 \mathrm{~ns}$.

The number of atomic radial distribution functions has been calculated to compare the strength of direct interactions between $\mathrm{EtOH}$, water and Tretinoin during the simulation time. The radial distribution function of Tretinoin around tube vs. distance is shown in Fig. 4.
Time(ps)

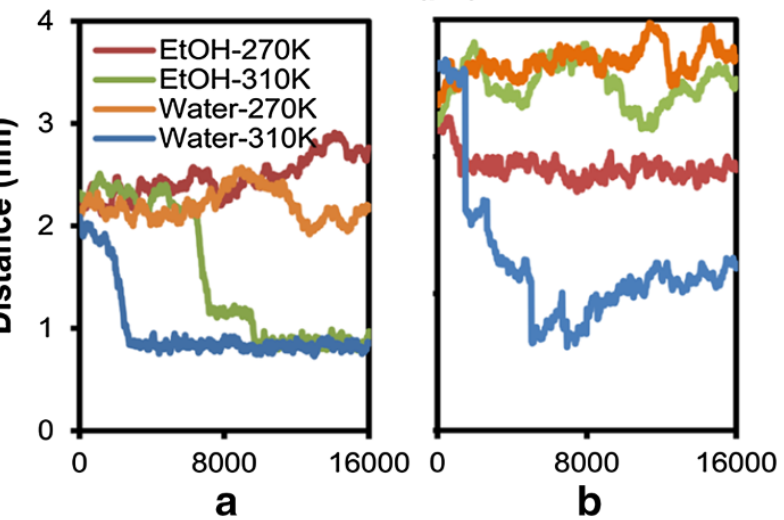

a

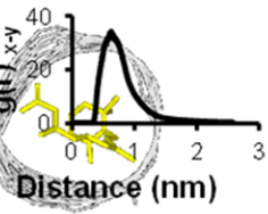

Distance $(\mathrm{nm})$

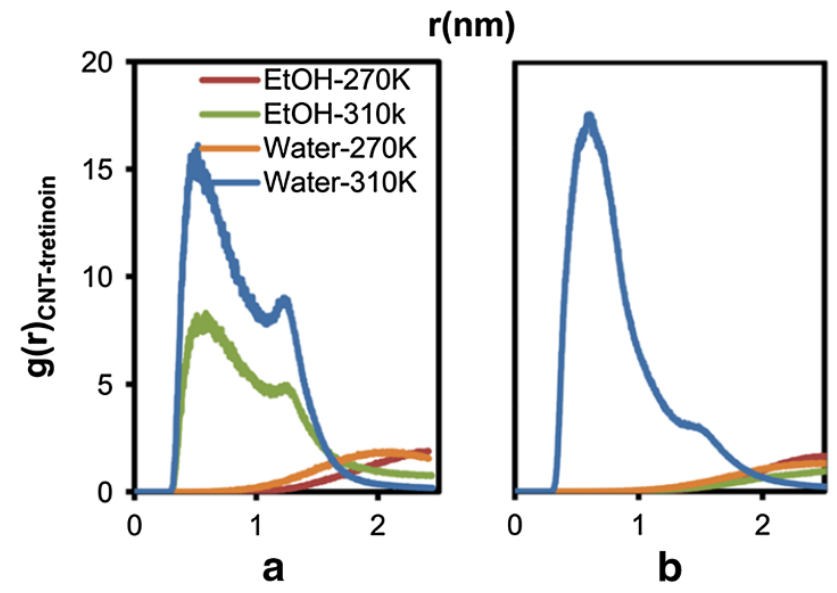

Fig. 4 Comparison of the radial distribution function of Tretinoin around tube $\mathbf{a}(8,5)$ and $\mathbf{b}(10,7)$ vs. distance

Results of Fig. 4a confirm more interaction of CNTTretinoin in the presence of water and $\mathrm{EtOH}$ at 310 that are in good agreement with results of Fig. 3a. It can be seen that interaction is increased in the water at $310 \mathrm{~K}$ (Fig. 4b) that is in consistency with Fig. $3 b$ results. The intensity of peak shows a strong interaction between CNT 


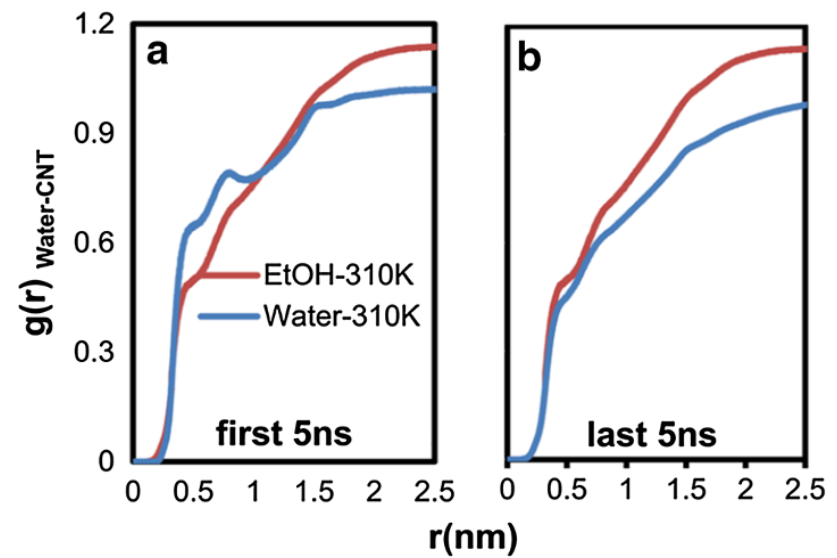

Fig. 5 Radial distribution function of water around CNT during the first and last $5 \mathrm{~ns}$

$(10,7)$-Tretinoin in water at $310 \mathrm{~K}$ that is also in agreement with Fig. 2 results. The sharp peak of Fig. $4 \mathrm{~b}$ compared to other curves reveals that there is a more ordered Tretinoin molecules near the CNT, indicative of better interaction of the atoms with time. The strength of the $g(r)$ profile between the CNT and Tretinoin in water is higher when compared to the $g(r)$ magnitude between the CNT and Tretinoin in EtOH co-solvent, particularly at higher temperatures. Thus, Tretinoin interacts more strongly with the CNT in water than cosolvent. The flat curves show a weak interaction at lower temperatures and in EtOH. The observed event was well supported by the previous studies, where the probability was found to increase exponentially as a function of the distance from the two ends of the CNT after encapsulation [30]. Note that a symmetric distribution of the $g(r)$ plot is expected if the sampling size is large enough, with a long enough simulation time.

In the radial distribution function of water around CNT, during the first and the last $5 \mathrm{~ns}$ (Fig. 5) which is before and after insertion, magnitude of RDF is directly proportional to water flow through CNT.

There are more water molecules near the CNT in the absence of EtOH before insertion (first $5 \mathrm{~ns}$ ), indicative of better solvation of the CNT in water. In the systems with co-solvent, there is a flow of water combined with a flow of co-solvent through nanotube before insertion that reduces water flow into the CNT as previously discussed [31]. Thus, water interacts more strongly with the CNT than with co-solvent, suggesting that accumulation of co-solvent around the CNT causes poor water flow into the CNT. The result of interaction during the last $5 \mathrm{~ns}$ (after insertion) indicates more water molecules near $\mathrm{CNT}$ in $\mathrm{EtOH}$ that no insertion is reported. The intensity of the first peak of system in the presence of water at $310 \mathrm{~K}$ decreased in the last $5 \mathrm{~ns}$, suggesting that insertion of Tretinoin into the

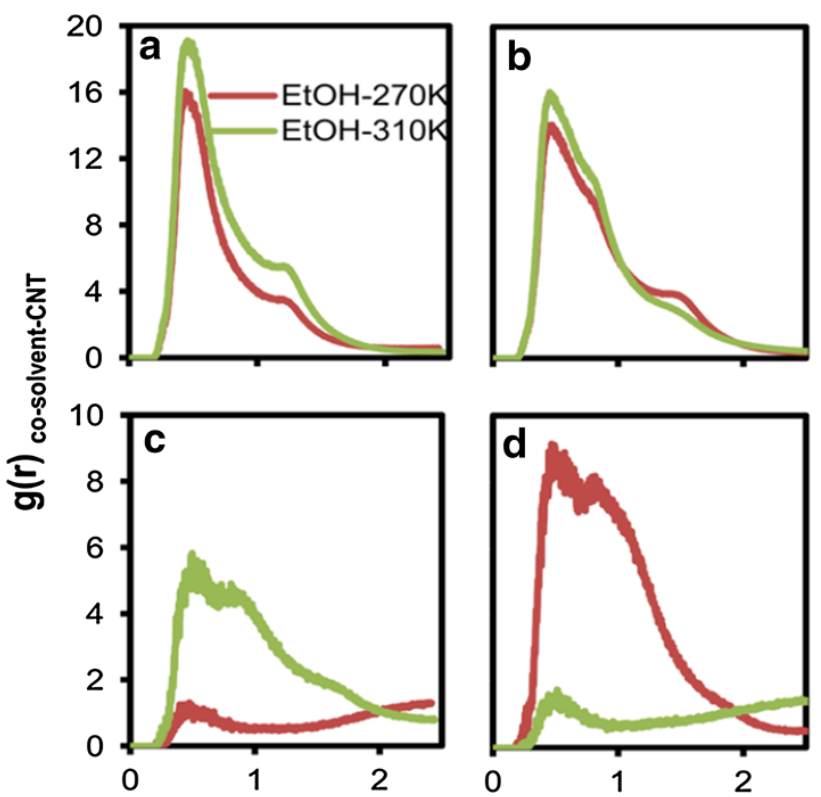

Fig. 6 Comparisons of the radial distribution function of co-solvent around a CNT $(8,5)$ and $\mathbf{b}$ CNT $(10,7)$ and around Tretinoin in the c CNT $(8,5)$ and d CNT $(10,7)$
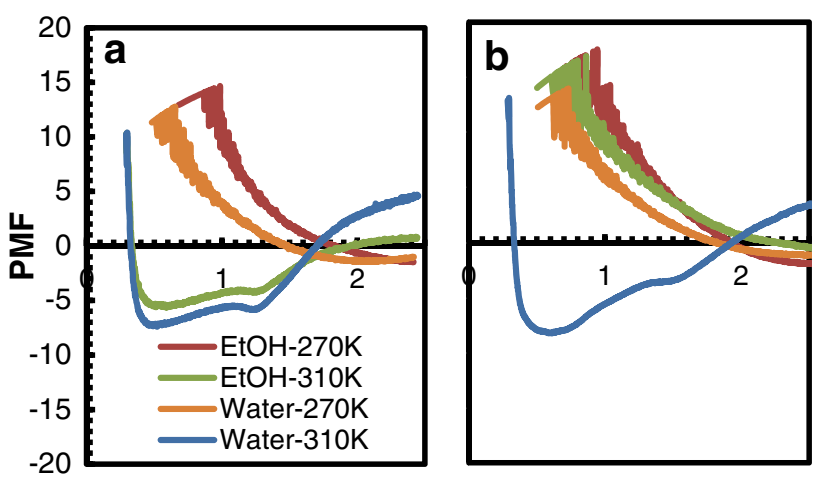

Fig. 7 Comparisons of the PMF of Tretinoin in a CNT $(8,5)$ and b CNT $(10,7)$

CNT causes poor solvation of the CNT that drives out water from the nanotube. Comparison of EtOH curves in Fig. 5a and $\mathrm{b}$ shows no special differences due to the lack of drug insertion. Totally, it can be concluded that water flow into nanotube helps Tretinoin insertion at the beginning of simulation and after insertion, water is expelled of nanotube and EtOH inhibits such insertion.

The radial distribution function of co-solvent around CNT vs. distance is shown in Fig. 6a and b. It can be seen that RDF is decreased more in lower temperatures and comparison of CNT size (Fig. 6a, b) reveals that by increasing CNT size, probability of finding CNT around co-solvent decreases. Figure $6 \mathrm{c}, \mathrm{d}$ presents existence probability of co-solvent around Tretinoin. The results show that at higher temperature $(310 \mathrm{~K})$, interaction 
Fig. 8 Comparison of the MSD of Tretinoin at 270 and $310 \mathrm{~K}$ in the presence of a CNT $(8,5)$ and $\mathbf{b}$ CNT $(10,7)$

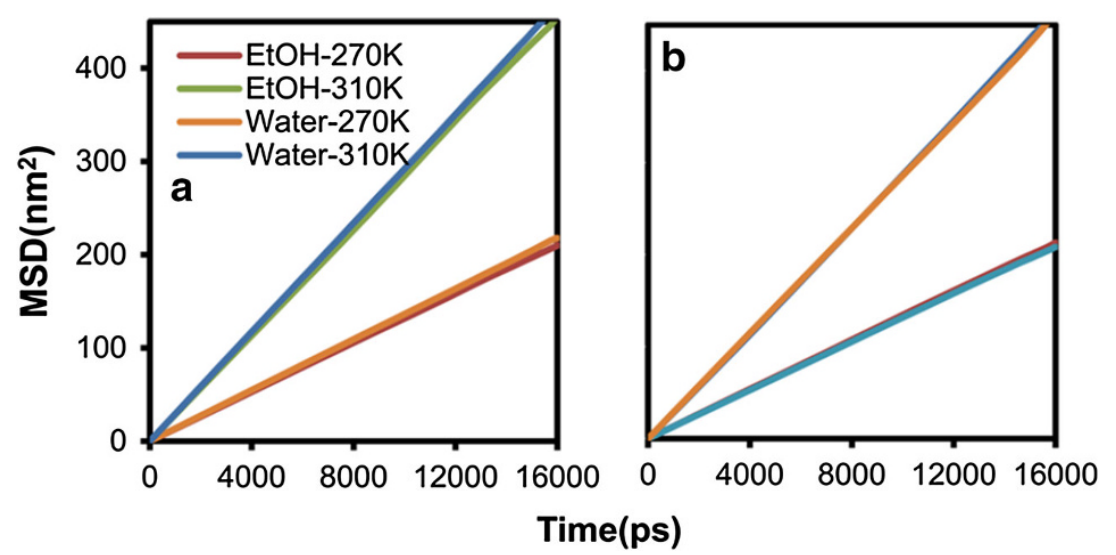

Table 4 Values of $D_{A}$ for CNT $(8,5)$ and $(10,7)$-water at 310 and $270 \mathrm{~K}$

\begin{tabular}{llll}
\hline No & $D_{A}$ & No & $D_{A}$ \\
\hline 1 & 0.002267 & 5 & 0.002183 \\
2 & 0.004867 & 6 & 0.004767 \\
3 & 0.002183 & 7 & 0.002217 \\
4 & 0.00475 & 8 & 0.004833
\end{tabular}

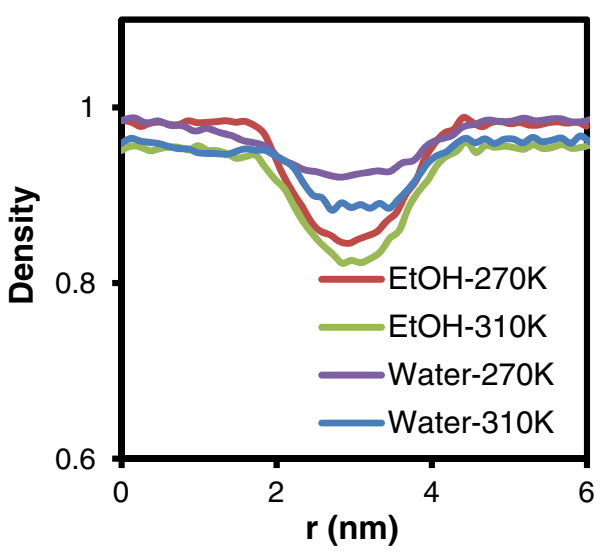

Fig. 9 Calculated density in different points in the box

between species decrease and according to Fig. 6b, there is more interaction between co-solvent-CNT than co-solvent Tretinoin. It confirms a flow of co-solvent through nanotube as previously suggested [31].

The profile of the PMF vs. the distance along the CNT is presented in Fig. 7. The obtained profile confirms the spontaneous encapsulation in the presence of water at $310 \mathrm{~K}$. The PMF decreases mainly when the drug reaches the tube edge and when insertion occurs, system becomes stable and PMF decreases.

The position of minima is $0.3-1.4 \mathrm{~nm}$ and $1.8-2.2 \mathrm{~nm}$ in the presence of water and $\mathrm{EtOH}$ respectively and the depth of minima increases in water and at higher temperatures in all solutions. Our results indicate stabilization of system in water compared to that in co-solvent. The depth of the PMF minimum increases as co-solvent is added and means that Tretinoin and CNT are in more favorable environment in water solution than EtOH; however, the minima is deeper in high temperatures. This is in good agreement with that yielded from the other drug delivery MD analysis investigated in recent years when the drug molecule moves within the CNT [31]. The energy data indicate clearly that drug molecule prefers to locate inside the CNT with a rather high energy barrier of $-5 \mathrm{kcal} \mathrm{mol}^{-1}$ to exit from the tube.

The average square distance, taken over all molecules and a measure of the average distance a molecule travels, gives us the mean square displacement (MSD). Figure 8 shows the MSD of Tretinoin for the CNT $(8,5)$ and CNT $(10,7)$ at two temperatures. This figure confirms less transport processes in the water solutions due to encapsulation process that occurs.

Values of $D_{A}$ obtained from MSD curves are presented in Table 4 for CNT $(8,5)$ and $\operatorname{CNT}(10,7)$-water at 310 and $270 \mathrm{~K}$, respectively. According to these values, Tretinoin molecule has more interaction with $\mathrm{CNT}(10,7)$ in water solution. The slope of MSD is lower for all systems at temperature $270 \mathrm{~K}$, indicating that the Tretinoin selfdiffusion constant $D_{A}$ increases as temperature is increased. In conjunction with previous studies [32], it is confirmed that a decrease in the CNT size and temperature leads to less steep MSD curve, implying less mobility and stability of systems and molecules.

Figure 9 shows density profile of water in four studied system and the average value of density of water was obtained below $1 \mathrm{~kg} \mathrm{~m}^{3}$. This suggested that the water molecules are rather sensitive to the environment, both when is free in the aqueous solution and when $\mathrm{EtOH}$ is added into the system or when the temperature is changed. Therefore, utilizing EtOH as the co-solvent affects the density of the water itself and decreases the density. Ethanol-water 
Fig. 10 The number of solvent (water/EtOH) molecules inside the CNT
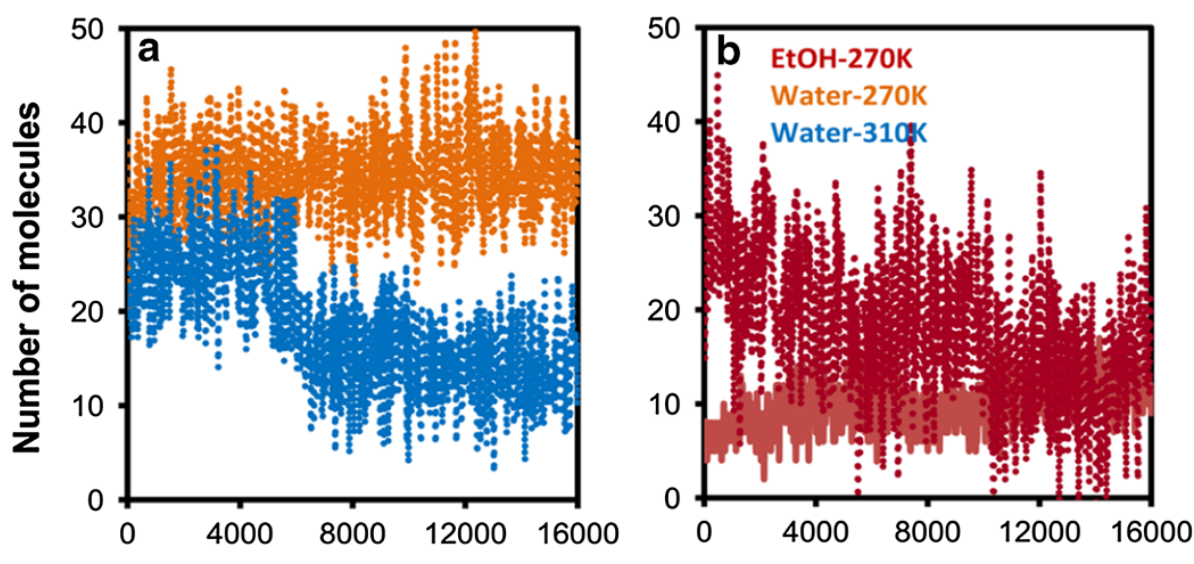

Time(ps)

mixtures have less volume than the sum of their individual components at the given fractions due to their ability to participate in strong hydrogen bonding. Results show that addition of EtOH and increase in temperature can reduce water density. The results were compared with the available experimental data as well as some theoretical models, overall indicating a good agreement [33].

To illustrate the interaction frequency between the CNT and $\mathrm{EtOH}$ in detail, we have calculated the numbers of atomic contacts of $\mathrm{CNT}(\mathrm{C})$ with $\mathrm{EtOH}$ and water (W) molecules, respectively. Here, we defined two molecules in contact if they are closer than $0.35 \mathrm{~nm}$. Figure 10 shows the number of solvent (water/EtOH) molecules inside the CNT during the course of simulation.

Figure 10a compares number of water molecules at two temperatures. Results of $310 \mathrm{~K}$ shows the number of water molecules increase at the beginning of simulation and then after $6 \mathrm{~ns}$, number of waters in the CNT decrease due to encapsulation of the drug into the nanotube. The curve of $270 \mathrm{~K}$ shows an inverse trend and over time, the number of water molecules in the tube increases and reaches a nearly constant value. It can be concluded that may be presence of more water at higher temperatures is the possible reason for encapsulation. Figure 10b compares number of water and EtOH molecules in the system $(0.2 \mathrm{M} \mathrm{EtOH})$ at $270 \mathrm{~K}$. Results show that the number of EtOH molecules increases and replaces water molecules. In this case, no encapsulation is observed and the wire of EtOH can be observed. It can be concluded that water insertion can be a driving force for drug encapsulation.

To examine characteristics of the Tretinoin movement from one end to another end throughout the tube, binding free energy profile from the MD simulations was evaluated using the LIE procedure successfully applied in previous works [26]. The results are given in Table 5.

As expected, the MD binding free energy is almost zero or positive when Tretinoin locates outside, and decreases
Table 5 Free energy values of systems obtained from LIE method

\begin{tabular}{|c|c|c|c|c|c|}
\hline \multirow[t]{2}{*}{ No } & \multicolumn{2}{|c|}{ Bounded $\left(\mathrm{kJ} \mathrm{mol}^{-1}\right)$} & \multicolumn{2}{|c|}{ free $\left(\mathrm{kJ} \mathrm{mol}^{-1}\right)$} & \multirow{2}{*}{$\begin{array}{l}\text { Free energy } \\
\left(\mathrm{kJ} \mathrm{mol}^{-1}\right)\end{array}$} \\
\hline & $\mathrm{L}-\mathrm{J}$ & VDW & $\mathrm{L}-\mathrm{J}$ & VDW & \\
\hline 1 & -66.5551 & -478.308 & -66.3175 & -478.512 & -0.0861 \\
\hline 2 & -89.8675 & -456.484 & -59.7638 & -465.139 & -13.6659 \\
\hline 3 & -66.9488 & -478.139 & -68.1889 & -475.035 & 0.1233 \\
\hline 4 & -77.1775 & -456.072 & -61.5375 & -462.338 & -6.7184 \\
\hline 5 & -66.4728 & -479.031 & -66.3175 & -478.512 & -0.1607 \\
\hline 6 & -149.926 & -435.275 & -59.7638 & -465.139 & -40.3029 \\
\hline 7 & -100.824 & -464.564 & -68.1889 & -475.035 & -11.082 \\
\hline 8 & -60.1905 & -464.261 & -61.5375 & -462.338 & -0.3076 \\
\hline
\end{tabular}

rapidly after the molecule enters the CNT. This result is in excellent agreement with those yielded from the previous MD investigations [34], when the drug molecule moves within the CNT. The binding free energy for Gemcitabine moving from one end to another end throughout the CNT was obtained and negative energy profiles in this case confirm that the drug molecule prefers to locate inside the CNT [33]. The energy data indicate clearly that drug molecule prefers to locate inside the CNT with a rather high energy barrier of $-40 \mathrm{~kJ} \mathrm{~mol}^{-1}$ to exit from the tube in water at $310 \mathrm{~K}$. As the $\mathrm{EtOH}$ molecules move from bulk into the CNT $(10,7)$, electrostatic interaction energies between CNT-Tretinoin are lost, which mainly come from the stronger dispersion interaction of EtOH with nanotube. In addition, the free energy analysis [by calculating the potential of mean force (PMF) and free energies of Table 5] also supports that the phenomenon of waterinduced insertion derives from the stronger effect of water with CNT than co-solvent. In Table 5, the most negative value of free energy is observed among system 1-4 in the presence of water at $310 \mathrm{~K}$ that confirms distance results of Fig. 5. There is another fact about the 5-8 systems. If the solvent dielectric constant increases, the Gibbs free energy 
values of the interaction between Tretinoin and CNT molecules decrease. The most negative value of free energy is for system 6 in agreement with above results which show more interaction of CNT-Tretinoin in water at $310 \mathrm{~K}$ due to the insertion of drug. In conclusion, investigated results show that $\mathrm{EtOH}$ has a reverse effect in insertion of drug because it has the most positive Gibbs free energy in this solvent. The MD results show that, for nanotubes of small diameters $<\operatorname{CNT}(10,7)$, the Tretinoin molecule is located on the outside of the nanotube in energetically most favorable conformations. For CNT (10, 7) or larger diameters, Tretinoin is to be located inside the tube.

\section{Conclusions}

In summary, a spontaneous insertion of an anticancer drug, Tretinoin into a $(10,7) \mathrm{CNT}$, is observed by MD simulation. This study shows the $\mathrm{VdW}$ interaction between the drug and CNT plays a significant role in this process. It was found that water molecules were repelled out of CNT during insertion. More importantly, the PMF profile obtained indicated that the PMF of system decreases after the encapsulation, which proves why spontaneous encapsulation occurs. The insertion of drug was studied for the smallest possible size of CNT. In conclusion, molecular dynamics simulations have indicated that Tretinoin could insert into the carbon nanotubes, with specific diameter in a water solute environment. Negative free energies, distance, RDF and PMF results confirmed localization of drug within CNT in water at $310 \mathrm{~K}$. Furthermore, heat capacity and energy results revealed that studied systems are more stable in lower temperatures and in water than the EtOH. Selfdiffusion coefficient of system is sensitive to the CNT and temperature. These results show that CNT $(10,7)$ is capable to be used in drug delivery process, and alcohols like EtOH inhibit the insertion process. This study has not addressed the concentration effect of $\mathrm{EtOH}$ (in normal range) on insertion. However, it is suggested to test larger size of nanotube during longer simulation time in future.

Acknowledgments The financial support of Damghan University is acknowledged. The authors thank Yasin Alipour for the computational support.

Conflict of interest The authors declare that they have no competing interests.

Open Access This article is distributed under the terms of the Creative Commons Attribution License which permits any use, distribution, and reproduction in any medium, provided the original author(s) and the source are credited.

\section{References}

1. Reed, A.E., Curtiss, L.A., Weinhold, F.: Intermolecular interactions from a natural bond orbital, donor-acceptor viewpoint. J. Chem. Rev 88, 899-926 (1988)

2. Yunpeng, Y., Xiaoyuan, C.: Integrin targeting for tumor optical imaging. Theranostics 1, 102-126 (2011)

3. Chen, X., Kis, A., Zettl, A., Bertozzi, C.R.: A cell nanoinjector based on carbon nanotubes. Proc. Natl. Acad. Sci. USA 104, 8218-8222 (2007)

4. Lin, J., Eder, M., Weinmann, S.: Behavioral counseling to prevent skin cancer: a systematic review for the U.S. Preventive Services Task Force. Ann. Intern. Med. 154, 190-201 (2011)

5. Narayanan, D., Saladi, R., Fox, J.: Ultraviolet radiation and skin cancer. Int. J. Dermatol. 49, 978-986 (2010)

6. Sersa, G., Miklavcic, D., Cemazar, M., Rudolf, Z., Pucihar, G., Snoj, M.: Electrochemotherapy in treatment of tumours. Eur. J. Surg. Oncol. 34, 232-240 (2008)

7. Joensuu, H.: Systemic chemotherapy for cancer: from weapon to treatment. Lancet Oncol. 9, 304 (2008)

8. Sanz, M.: Treatment of acute promyelocytic leukemia. Hematol. Am. Soc. Hematol. Educ. Progr. 15, 147-155 (2006)

9. Hesabi, M., Hesabi, M.: The interaction between carbon nanotube and skin anti-cancer drugs: a DFT and NBO approach. J. Nanostruct. Chem. 3, 22 (2013)

10. Seram, H.A., Coorey, R.V.: Study of the effect of temperature on water permeation through Carbon nanotubes subjected to an induced pressure gradient: a molecular dynamics simulation. Proc. Tech. Sess. 27, 17-25 (2011)

11. Dalili Mansour, N., Zare, K., Elsagh, A.: Theoretical study of drug delivery ability of carbon nanotube. J. Phys. Theor. Chem. 7, 15-21 (2010)

12. Kiruta, N.V., Dyachkov, E.P., Dyachkov, P.N.: Supra molecular complexes of carbon nanotubes with doxorubicin and poly (Ethylen glycol) studied using the molecular docking and dynamic methods. Comp. Model. New. Technol. 15, 16-22 (2011)

13. Dalili Mansour, N., Zare, K., Elsagh, A.: Theoretical study of drug delivery ability of carbon nanotube. J. Phys. Theo. Chem. 7, 15-21 (2010)

14. Cornell, W.D., Cieplak, P., Bayly, C.I., Gould, I.R., Merz Jr, K.M., Ferguson, D.M., Spellmeyer, D.C., Fox, T., Caldwell, J.W., Kollman, P.A.: A second generation force field for the simulation of proteins, nucleic acids, and organic molecules. J. Am. Chem. Soc. 117, 5179-5197 (1995)

15. Dewar, M.J.S., Zoebisch, E.G., Healy, E.F., Stewart, J.J.P.: Development and use of quantum mechanical molecular models. 76. AM1: a new general purpose quantum mechanical molecular model. J. Am. Chem. Soc. 107, 3902-3909 (1985)

16. Schuttelkopf, A.W., van Aalten, D.M.F.: PRODRG: a tool for high-throughput crystallography of protein-ligand complexes. Acta Crystallogr. 60, 1355-1363 (2004)

17. Frey, J.T., Doren, D.J.: University of Delaware, Newark DE (2011). http://turin.nss.udel.edu/research/tubegenonline.html

18. Chaban, V.V., Kalugin, D.N.: Structure and dynamics in methanol and its lithium ion solution confined by carbon nanotubes. J. Mol. Liq. 145, 145-151 (2009)

19. Kalugin, O.N., Kalugin, D.N.: Liquid dimethyl sulphoxide confined by carbon nanotubes. J. Mol. Liq. 151, 113-116 (2010)

20. Soares, T., Daura, X., Oostenbrink, C., Smith, L.J., van Gunsteren, W.F.: Validation of the GROMOSE force field parameter set 45 A3 against nuclear magnetic resonance data of hen egg lyzozyme. J. Biomol. NMR 30, 407-422 (2004)

21. Walther, J.H., Jaffe, R., Halicioglu, T., Koumoutsakos, P.: Carbon nanotubes in water: structural characteristics and energetics. J. Phys. Chem. B 105, 9980-9987 (2001) 
22. Van Der Spoel, D.: GROMACS: fast, flexible, and free. J. Comput. Chem. 26, 1701-1718 (2005)

23. Darden, T., York, D., Pedersen, L.: Particle mesh Ewald-an N. $\log (\mathrm{N})$ method for Ewald sums in large systems. J. Chem. Phys. 98, 10089 (1993)

24. Yakubovich, A.V., Solovyov, I.A., Solovyova, A.V., Greiner, W.: Phase transitions in polypeptides: analysis of energy fluctuations. J. Eur. Phys. D 51, 25-32 (2009)

25. Allen, M.P., Tildesley, D.J.: Computer simulations of liquids. Oxford Science Publications, Oxford (1987)

26. Wang, W., Wang, J.: Kollman PA: What determines the van der Waals coefficient $\beta$ in the LIE (Linear Interaction Energy) method to estimate binding free energies using molecular dynamics simulations? PROTEINS Struct. Funct. Genet. 34, 395-402 (1999)

27. Mehrnejad, F., Ghahremanpour, M.M., Khadem-Maaref, M., Doustdar, F.: Effects of osmolytes on the helical conformation of model peptide: molecular dynamics simulation. J. Chem. Phys. 134, 035104 (2011)

28. Rauf, M.A., Hisaindee, S., Graham, J.P., Nawaz, M.: Solvent effects on the absorption and fluorescence spectra of $\mathrm{Cu}(\mathrm{II})$-phthalocyanine, and DFT calculations. J. Mol. Liq. 168, 102-109 (2012)

29. Terzyk, A.P., Pacholczyk, A., Wisniewski, M., Gauden, P.A.: Enhanced adsorption of paracetamol on closed carbon nanotubes by formation of nanoaggregates: carbon nanotubes as potential materials in hot-melt drug deposition-experiment and simulation. J. Colloid Interface Sci. 376, 209-216 (2012)

30. Terzyk, A.P., Wisniewski, M., Dulska, K., Bielicka, A., Gauden, P.A., Furmaniak, S., Werengowska-Ciecwierz, K.: Carbon nanotubes as potential material for drug delivery-experiment and simulation. Adsorption 19, 269-272 (2013)

31. Xiu, P., Yang, Z.X., Zhou, B.: Urea-induced drying of hydrophobic nanotubes: comparison of different Urea models. J. Phys. Chem. B 115, 2988-2994 (2011)

32. Yang-Hong, W., Yu-Juan, C., Ling, B., Xue-Mei, L., Shuan-Shi, F.: Molecular dynamics simulation of $\mathrm{CH} 4$ hydrate decomposition in the presence of poly (2-ethyl2-oxazoline). Acta Phys. Chim. Sin. 28, 1683-1690 (2012)

33. Khattab, I.S., Bandarkar, F., Abolghasemi Fakhree, M.M., Jouyban, A.: Density, viscosity and surface tension of water + ethanol mixtures from 293 to $323 \mathrm{~K}$. Korean J. Chem. Eng 29, 812-813 (2012)

34. Arsawanga, U., Saengsawangb, O., Rungrotmongkol, K., Sornmeea, P., Wittayanarakul, K., Remsungnend, T., Hannongbuab, S.: How do carbon nanotubes serve as carriers for gemcitabine transport in a drug delivery system? J. Mol. Graph. Model. 29, 591-596 (2011) 